\title{
The Rate of Convergence to Perfect Competition of Matching and Bargaining Mechanisms
}

\author{
Artyom Shneyerov \\ CIREQ, CIRANO and Concordia University, Montréal, Canada \\ Adam Chi Leung Wong* \\ University of British Columbia, Vancouver, Canada
}

November, 2006

This version: August, 2008

\begin{abstract}
We study the steady state of a market with incoming cohorts of buyers and sellers who are matched pairwise and bargain under private information. We first consider generalized random-proposer take-it-or-leave-it offer games (GRP TIOLI games). This class of games includes a simple random-proposer TIOLI game, but also many other interesting bargaining games. A friction parameter is $\tau$, the length of the time period until the next meeting. We find that as $\tau \rightarrow 0$, all market equilibria converge to the Walrasian limit, at the fastest possible rate $O(\tau)$ among all bargaining mechanisms. Some important bargaining games not in this class may have non-convergent market equilibria. This is the case for the $k$-double auction: we find that there are equilibria that converge at a linear rate, those that converge at a slower rate or even not converge at all.
\end{abstract}

Keywords: Matching and Bargaining, Search, Double Auctions, Foundations for Perfect Competition, Rate of Convergence

JEL Classification Numbers: C73, C78, D83.

\section{Introduction}

A number of papers in the literature on dynamic matching and bargaining have shown that, as frictions vanish, equilibria converge to perfect competition. ${ }^{1}$ But it is also important to

\footnotetext{
${ }^{*}$ We thank Mike Peters, Mark Satterthwaite, and seminar participants at Northwestern, SFU and UWO for helpful comments. We thank SSHRC for financial support made available through grants $12 \mathrm{R} 27261$ and 12R27788. This version of the paper was completed when the first author was visiting the Center for Mathematical Studies in Economics and Management Science at Kellogg School of Management, Northwestern University. Its warm hospitality is gratefully acknowledged.

${ }^{1}$ Papers that address convergence include Rubinstein and Wolinsky (1985), Gale (1986), Gale (1987), Rubinstein and Wolinsky (1990), Mortensen and Wright (2002), and, with private information, Butters (1979), Wolinsky (1988), De Fraja and Sakovics (2001), Serrano (2002), Moreno and Wooders (2002), Lauermann (2006a), Satterthwaite and Shneyerov (2007), Atakan (2008).
} 
know how rapidly the equilibria converge. To our knowledge, this question has not been addressed in the literature.

In contrast, the rate of convergence to efficiency has been the focus of the literature on static double auctions. It is important to know how large $n$ needs to be so that we can call a double auction with $n$ buyers and sellers approximately competitive. Rustichini, Satterthwaite, and Williams (1994) show robust convergence of double-auction equilibria in the symmetric class at the fast rate $O(1 / n)$ for the bid/offer strategies and the superfast rate $O\left(1 / n^{2}\right)$ for the ex-ante traders' welfare, where $n$ is the number of traders in the market. ${ }^{2}$ Moreover, the double auction converges at the rate that is fastest among all incentive-compatible and individually rational mechanisms (Satterthwaite and Williams (2002); Tatur (2005)). Cripps and Swinkels (2005) substantially enrich the model by allowing correlation among bidders' valuations, and show convergence at the rate $O\left(1 / n^{2-\varepsilon}\right)$, where $\varepsilon>0$ is arbitrarily small. ${ }^{3}$

For a dynamic matching and bargaining market, the question of how small frictions need to be for equilibria to be approximately competitive is equally important. In this paper, we fill this gap by proving a rate of convergence result for a decentralized model of trade. We study the steady state of a market with incoming cohorts of buyers and sellers who are randomly matched pairwise and bargain without knowing each other's reservation value. The model is in discrete time and shares several features with the model in Satterthwaite and Shneyerov (2007). Exactly as in that paper, a friction parameter is $\tau$, the length of the time period until the next meeting. There are per-period participation costs, $\tau \kappa_{B}$ for buyers and $\tau \kappa_{S}$ for sellers. There is also time discounting at the instantaneous rate $r .{ }^{4}$

Our model is different from Satterthwaite and Shneyerov (2007) in that we consider pairwise matching and general bargaining games. (Satterthwaite and Shneyerov (2007) restrict attention to auctions.) Specifically, our baseline bargaining protocol is a generalization of a simple random-proposer take-it-or-leave-it (TIOLI) protocol in which each trader is chosen as a proposer with certain probability. ${ }^{5}$ We consider bargaining games that generalize the following two basic intuitive properties of this simple game. First, each trader has some bargaining power in the sense that he or she can offer the terms of trade with some probability. Second, trade is voluntary in the sense that ex-post, the parties may walk away from the deal if the price is not satisfactory. Third, money changes hands only if the good does. Specifically, for the first property we require that each trader has a strategy guaranteeing that he or she is winds up being a proposer in a TIOLI node of the extensive form of the game with some probability, regardless of the strategy used by the other trader. For the second property, we require that the outcome of the game is ex-post individually rational.

\footnotetext{
${ }^{2}$ See also Gresik and Satterthwaite (1989), Satterthwaite and Williams (1989), Satterthwaite (1989), Williams (1991).

${ }^{3}$ Reny and Perry (2006) allow interdependent values and show that it is almost efficient and almost fully aggregates information as $n \rightarrow \infty$, but do not address the rate of convergence issue.

${ }^{4}$ Satterthwaite and Shneyerov (2007) also discuss the rate of convergence to perfect competition, and the relevance of making $\tau$ small. However, there is no general rate of convergence result, only within a class of full-trade equilibria. They also discuss an interpretation of the inverse of $\tau$ as a measure of local market size. We do not repeat these discussions here.

${ }^{5}$ The existence and properties of equilibria with this protocol are studied in Shneyerov and Wong (2007), but in a continuous time setting as in Mortensen and Wright (2002).
} 
We call this class of games generalized random-proposer TIOLI games, or, in an abbreviated form, GRP TIOLI games. The simple random-proposer TIOLI game described above is obviously in this class. Many other interesting bargaining games are also GRP TIOLI: 6

- Finitely repeated simple random-proposer TIOLI game that may be exogenously terminated with probability $\beta \in(0,1)$ after each rejected offer.

- Repeated-offer bargaining games in which the seller is chosen as a proposer with probability $\alpha \in(0,1)$, and the buyer is chosen with the complementary probability. The proposer then makes a number of sequential offers to the responder. After each rejected offer, the game may be exogenously terminated with probability $\beta \in(0,1)$.

- Alternate-offer bargaining games in which the first proposer is chosen to be the seller with probability $\alpha \in(0,1)$ and the buyer with probability $1-\alpha$. The traders alternate making proposals for several rounds. The last proposer is different from the proposer chosen in the first round. Once again, the game may be terminated with probability $\beta \in(0,1)$ after each rejected offer.

The above games are GRP TIOLI because each trader, by rejecting all offers and making unacceptable offers until the final round, guarantees that he is a TIOLI proposer with certain probability.

We derive explicit bounds on the inefficiency of any nontrivial market equilibrium for GRP TIOLI bargaining mechanisms. ${ }^{7}$ These bounds imply that inefficiency vanishes at a linear rate in $\tau$. Thus all (nontrivial) equilibria converge rapidly to the Walrasian benchmark. Using the notion of worst-case asymptotic optimality similar to Satterthwaite and Williams (2002), we show that the GRP TIOLI mechanisms are worst-case asymptotically optimal: in terms of the welfare, the rate of convergence cannot be faster under any other bargaining mechanism. ${ }^{8}$

With the exception of Lauermann (2006b) and Atakan (2008), all papers in the matching and bargaining literature that we are aware of consider specific bargaining protocols. Lauermann (2006b) considers a general class of bargaining games. He shows that equilibria converge to perfect competition provided that the sequence of outcomes of the game satisfies certain conditions. Our conditions are different in that they are conditions on the primitives of the game. Atakan (2008) provides an important extension of the results of Satterthwaite and Shneyerov (2007) to multiple units. Atakan (2008) allows the players to propose general bargaining mechanisms, and shows that they can do no better than use simple TIOLI offer strategies. With this justification, he confines the actual analysis to the simple random-proposer TIOLI games.

\footnotetext{
${ }^{6}$ These examples are discussed for example in Ausubel, Cramton, and Deneckere (2002).

${ }^{7} \mathrm{~A}$ trivial, uninteresting equilibrium in which none of the traders enter always exists.

${ }^{8}$ The notion of the "worst case" here is actually weaker because we only look at the most unfavorable equilibrium; all our results are for fixed distributions of traders' types. The definition of the worst case in Satterthwaite and Williams (2002) involves also searching for the most unfavorable distributions of traders' types.
} 
Ruled out by the GRP TIOLI assumption are bargaining games in which only one side is given all the bargaining power, i.e. only one of the traders can be a proposer. ${ }^{9}$ Also ruled out are games without TIOLI nodes, as would be the case for $k$-double auctions. We study convergence properties of market equilibria with k-double auctions and find that equilibria can be either convergent at a linear rate, convergent at a slower-than-linear rate or even divergent. This result can be compared to the findings in Serrano (2002). In a dynamic setting, Serrano (2002) studies a mechanism that in some respects resembles a double auction (the set of bids is restricted to be a finite grid) and finds that "as discounting is removed, equilibria with Walrasian and non-Walrasian features persist". ${ }^{10}$ Serrano points out, however, that "after removing the finite sets of traders' types and of allowed prices, the present model confirms Gale's one-price result and has a strong Walrasian flavor". We, on the other hand, find that in our model, non-convergent equilibria exist even if the bargaining protocol is the "unrestricted" double auction.

\section{The Baseline Model}

The agents are potential buyers and sellers of a homogeneous, indivisible good. Each buyer has a unit demand, while each seller has a unit supply. All traders are risk neutral. Potential buyers are heterogeneous in their valuations (or types) $v$ of the good. Potential sellers are also heterogeneous in their costs (or types) $c$ of providing the good. The buyers draw their types i.i.d. from some distribution $F$ and the sellers draw their types i.i.d. from some distribution $G$. For simplicity, we assume that the supports of these distributions are $[0,1]$. Each trader's type remains the same over time. We index time periods by $t=\cdots,-1,0,1, \cdots$; each time period has length $\tau>0$. The instantaneous discount rate is $r \geq 0$, and the corresponding discount factor is $R_{\tau}=e^{-r \tau}$. Each period consists of the following stages.

- The mass $b$ of potential buyers and the mass $s$ of potential sellers are born. The new-born buyers draw their types i.i.d. from the distribution $F$ and the new-born sellers draw their types i.i.d. from the distribution $G$.

- Entry (or participation, or being active): The new-born potential buyers and sellers decide whether to enter the market. Those who enter together with the current pools of traders in the market compose the set of active traders.

- The active buyers and sellers incur participation costs $\tau \kappa_{B}$ and $\tau \kappa_{S}$ respectively.

- The active buyers and sellers are randomly matched in pairs. The mass of the matches is given by a matching function $M(B, S)$, where $B$ and $S$ are the masses of active buyers and active sellers currently in the market. The probability $\ell_{B}$ that a buyer is matched is equal to $M(B, S) / B$, and he is equally likely to meet any active

\footnotetext{
${ }^{9}$ Lauermann (2008) shows convergence to perfect competition in a model where one side is given all bargaining power. As in Satterthwaite and Shneyerov (2008), he assumes that there is no participation cost, but there is an exogenous exit rate of traders.

${ }^{10}$ This simplified bargaining mechanism was introduced in Wolinsky (1990) and also used in Blouin and Serrano (2001).
} 
seller. Symmetrically, the seller's matching probability is $\ell_{S}=M(B, S) / B$ and she is equally likely to meet any active buyer. ${ }^{11}$ The matching is anonymous.

- The matched buyers and sellers bargain. We assume that bargaining is instantaneous, and consider general bargaining protocols. If a type $v$ buyer and a type $c$ seller trade at a price $p$, then they leave the market with utilities $v-p$ and $p-c$. If the bargaining breaks down, both traders remain in the market.

Assumption 1 (matching function) The matching function $M$ is continuous on $\mathbb{R}_{+}^{2}$, nondecreasing in each argument, exhibits constant returns to scale (i.e. is homogeneous of degree one), and satisfies $M(0, S)=M(B, 0)=0$.

Let $\zeta \equiv B / S$ be the steady-state ratio of the mass of buyers to the mass of sellers (or market tightness), and define $m(\zeta) \equiv M(\zeta, 1)$. Since the matching technology is constant returns to scale, the matching probabilities for buyers and sellers are

$$
\ell_{B}(\zeta) \equiv \frac{m(\zeta)}{\zeta}, \quad \ell_{S}(\zeta) \equiv m(\zeta)
$$

Note that $\ell_{B}$ and $\ell_{S}$ are continuous on $\mathbb{R}_{++}$, and respectively nonincreasing and nondecreasing functions of $\zeta$.

Define

$$
K(\zeta)=\frac{\kappa_{B}}{\ell_{B}(\zeta)}+\frac{\kappa_{S}}{\ell_{S}(\zeta)}
$$

The function $K(\zeta)$ can be interpreted as the participation costs incurred by a pair of traders over the time period of length $\tau=1$, inflated by their probabilities of matching $\ell_{B}(\zeta)$ and $\ell_{S}(\zeta)$.

Our equilibrium notion parallels that of Satterthwaite and Shneyerov (2007), so we skip many details elucidated there and focus on the differences due to the fact that we are considering general bargaining mechanisms. We assume that the market is in a steady state and denote the distribution of active buyer and seller types as $\Phi$ and $\Gamma$. We denote the supports of these distributions as $A_{B}$ and $A_{S}$. We only consider equilibria with entry, i.e. those in which the steady-state masses $B$ and $S$ of active buyers and sellers are positive. Denote as $W_{B}(v)$ and $W_{S}(c)$ the beginning-of-period market utilities of type $v$ buyer and

\footnotetext{
${ }^{11}$ We conjecture that such a matching process can arise in the limit of finite economies. Let $\mathbb{G}_{L}^{n_{B}, n_{S}}$ be the set of all bilateral matching graphs with $n_{B}$ buyer nodes, $n_{S}$ seller nodes and $L$ edges. All the edges are assumed to be nonadjacent. Let $\mathbb{P}_{L}^{n_{B}, n_{S}}$ be the probability measure on $\mathbb{G}_{L}^{n_{B}, n_{S}}$ that assigns equal probability to each graph in $\mathbb{G}_{L}^{n_{B}, n_{S}}$. In this stochastic matching model, (i) the probability of matching is $L / n_{B}$ for the buyers and $L / n_{S}$ for the seller, and (ii) conditional on matching, each buyer is equally likely to be matched with any seller. Our matching technology can be understood in this framework, as arising in the limit as $n \rightarrow \infty$ of the measures $\mathbb{P}_{M\left(n_{B}, n_{S}\right)}^{n_{B}, n_{S}}$ with $n_{B}$ approximately equal to $n \cdot B$ and $n_{S}$ approximately equal to $n \cdot S$. If the matching function exhibits constant returns to scale, it is easy to see that a buyer's probability of matching $\ell_{B}=M\left(n_{B}, n_{S}\right) / n_{B} \approx M(B, S) / B$, and similarly $\ell_{S} \approx M(B, S) / B$. Moreover, each buyer continues to be equally likely matched with any seller.
} 
type $c$ seller. Our twin assumptions of the continua of traders and anonymous matching imply that the reservation prices in the bargaining game do not depend on the strategies used in the game and are given by

$$
\tilde{v}(v)=v-R_{\tau} W_{B}(v), \quad \tilde{c}(c)=c+R_{\tau} W_{S}(c) .
$$

Following Satterthwaite and Shneyerov (2007), we will call these the dynamic types of buyers and sellers. The market distributions of $\tilde{v}(v)$ and $\tilde{c}(c)$ are denoted as $\tilde{\Phi}$ and $\tilde{\Gamma}$.

In each meeting, for given types $c$ and $v$, a (direct) bargaining mechanism determines the trading probability $q(v, c)$ and the expected payment $t(v, c)$ made by the buyer to the seller. Given that the opponent types are drawn from the market distributions $\Phi$ and $\Gamma$, the associated interim probabilities and payments are

$$
\begin{aligned}
q_{B}(v) & =\int q(v, c) d \Gamma(c), \quad q_{S}(c)=\int q(v, c) d \Phi(v), \\
t_{B}(v) & =\int t(v, c) d \Gamma(c), \quad t_{S}(c)=\int t(v, c) d \Phi(v) .
\end{aligned}
$$

We assume that the mechanism is incentive compatible: the expected payoffs to the traders, over and above their discounted market utilities $R_{\tau} W_{B}(v)$ and $R_{\tau} W_{S}(c)$, are maximal under truthful reporting:

$$
\begin{aligned}
\tilde{v}(v) q_{B}(v)-t_{B}(v) & =\max _{v^{\prime} \in[0,1]} \tilde{v}(v) q_{B}\left(v^{\prime}\right)-t_{B}\left(v^{\prime}\right), \\
t_{S}(c)-\tilde{c}(c) q_{S}(c) & =\max _{c^{\prime} \in[0,1]} t_{S}\left(c^{\prime}\right)-\tilde{c}(c) q_{S}\left(c^{\prime}\right) .
\end{aligned}
$$

We assume that money changes hands only through trade. We also assume that whenever trade occurs, the price must be acceptable to both traders. In other words, whenever $q(v, c)>0$ so that the expected trading price $p(v, c)$ is defined, it must satisfy the ex-post individual rationality condition

$$
p(v, c) \in[\tilde{c}(c), \tilde{v}(v)] .
$$

Given that participation is voluntary, the market utilities $W_{B}(v)$ and $W_{S}(c)$ must satisfy the recursive equations

$$
\begin{gathered}
W_{B}(v)=\max \left\{\ell_{B}\left[v q_{B}(v)-t_{B}(v)\right]+\left(1-\ell_{B} q_{B}(v)\right) R_{\tau} W_{B}(v)-\tau \kappa_{B}, 0\right\}, \\
W_{S}(c)=\max \left\{\ell_{S}\left[c q_{S}(c)-t_{S}(c)\right]+\left(1-\ell_{S} q_{S}(c)\right) R_{\tau} W_{S}(c)-\tau \kappa_{S}, 0\right\} .
\end{gathered}
$$

The sets of active buyers' and sellers' types, $A_{B}$ and $A_{S}$, are the closures of the sets $\left\{v \in[0,1]: W_{B}(v)>0\right\}$ and $\left\{c \in[0,1]: W_{S}(c)>0\right\}$, i.e. we include the marginal traders who are indifferent between entering or not. The steady-state assumption implies the following mass balance conditions for $v \in A_{B}, c \in A_{S}$ :

$$
b \cdot d F(v)=B \ell_{B}(\zeta) q_{B}(v) \cdot d \Phi(v), \quad s \cdot d G(c)=S \ell_{S}(\zeta) q_{S}(c) \cdot d \Gamma(c) .
$$

These equations complete the description of the market equilibrium constellation $E=$ $\left(q_{B}, q_{S}, t_{B}, t_{S}, W_{B}, W_{S}, B, S, \Phi, \Gamma\right)$. 


\section{Basic Properties of Equilibria}

Our first result shows that, for any bargaining protocol, the sets $A_{B}$ and $A_{S}$ must be intervals, and derives the properties of the dynamic type functions $\tilde{v}, \tilde{c}$ and equilibrium trading probabilities $q_{B}, q_{S}$.

Lemma 1 For any bargaining protocol, the sets of active trader types are intervals: $A_{B}=$ $[\underline{v}, 1]$ and $A_{S}=[0, \bar{c}]$. The trading probability $q_{B}(v)$ is strictly positive and nondecreasing in $v$ on $A_{B}$, while $q_{S}(c)$ is strictly positive and nonincreasing in $c$ on $A_{S}$. Moreover,

$$
\begin{array}{cl}
W_{B}(v) & =\int_{\underline{v}}^{v} \frac{\ell_{B} q_{B}(x)}{1-R_{\tau}+R_{\tau} \ell_{B} q_{B}(x)} d x \quad \text { for all } v \in[\underline{v}, 1], \\
W_{S}(c) & =\int_{c}^{\bar{c}} \frac{\ell_{S} q_{S}(x)}{1-R_{\tau}+R_{\tau} \ell_{S} q_{S}(x)} d x \quad \text { for all } c \in[0, \bar{c}] .
\end{array}
$$

The responding strategies $\tilde{v}$ and $\tilde{c}$ are absolutely continuous and nondecreasing. Their slopes are

$$
\begin{gathered}
\tilde{v}^{\prime}(v)=\frac{1-R_{\tau}}{1-R_{\tau}+R_{\tau} \ell_{B} q_{B}(v)} \quad\left(\text { a.e. } v \in A_{B}\right), \\
\tilde{c}^{\prime}(c)=\frac{1-R_{\tau}}{1-R_{\tau}+R_{\tau} \ell_{S} q_{S}(c)} \quad\left(\text { a.e. } c \in A_{S}\right) .
\end{gathered}
$$

Proof. We prove the results for buyers only; the argument for the sellers is parallel. We begin by noting that the incentive compatibility condition (2) and the recursive equation (5) together imply that for a participating type $v \in A_{B}$

$$
W_{B}(v)=\max _{v^{\prime} \in[0,1]}\left\{\ell_{B}\left[v q_{B}\left(v^{\prime}\right)-t_{B}\left(v^{\prime}\right)\right]+\left(1-\ell_{B} q_{B}\left(v^{\prime}\right)\right) R_{\tau} W_{B}(v)-\tau \kappa_{B}\right\} .
$$

Condition (12) implies for $v \in A_{B}$ :

$$
W_{B}(v)=\max _{v^{\prime} \in[0,1]} \frac{\ell_{B}\left[v q_{B}\left(v^{\prime}\right)-t_{B}\left(v^{\prime}\right)\right]-\tau \kappa_{B}}{1-R_{\tau}+R_{\tau} \ell_{B} q_{B}\left(v^{\prime}\right)} .
$$

For the type that have chosen not to become active, the r.h.s. of the above inequality is less than or equal to 0 and $W_{B}(v)=0$. We therefore can write for any $v \in[0,1]$ :

$$
W_{B}(v)=\max _{\chi \in\{0,1\}, v^{\prime} \in[0,1]} \chi \cdot\left\{\frac{\ell_{B}\left[v q_{B}\left(v^{\prime}\right)-t_{B}\left(v^{\prime}\right)\right]-\tau \kappa_{B}}{1-R_{\tau}+R_{\tau} \ell_{B} q_{B}\left(v^{\prime}\right)}\right\} .
$$

As a maximum of the set of nondecreasing and affine functions, $W_{B}$ is continuous, convex and therefore absolutely continuous and differentiable almost everywhere on $[0,1]$. The Envelope Theorem (Theorem 2 in Milgrom and Segal (2002)) then implies

$$
W_{B}^{\prime}(v)=\frac{\ell_{B} q_{B}(v)}{1-R_{\tau}+R_{\tau} \ell_{B} q_{B}(v)} \text { a.e. on } A_{B},
$$

and (10) must also hold. Since $q_{B}(v)>0$ for $v \in A_{B}$, and $W_{B}$ is absolutely continuous, it must be increasing on $A_{B}$. Moreover, $A_{B}$ must be an interval $[\underline{v}, 1]$, and (8) follows. Finally, the convexity of $W_{B}$ implies that $q_{B}$ is nondecreasing. Q.E.D. 
Our interest is in small frictions, i.e. small $\tau>0$ : how far are the equilibrium market utilities $W_{B}(v)$ and $W_{S}(c)$ from their Walrasian counterparts? We define the competitive, or Walrasian, price $p^{*}$ as the price that clears the flows of the arriving cohorts:

$$
b\left[1-F\left(p^{*}\right)\right]=s G\left(p^{*}\right),
$$

and define traders' Walrasian utilities in the usual manner, as

$$
W_{B}^{*}(v)=\max \left\{v-p^{*}, 0\right\}, \quad W_{S}^{*}(c)=\max \left\{p^{*}-c, 0\right\} .
$$

The total Walrasian surplus is then

$$
W^{0 *}=b \int_{p^{*}}^{1}\left(v-p^{*}\right) d F(v)+s \int_{0}^{p^{*}}\left(p^{*}-c\right) d G(c) .
$$

Since the sets of active trader types are intervals, we call $\underline{v}$ and $\bar{c}$ the marginal participating types. The buyers (sellers) of type $\underline{v}(\bar{c})$, who are indifferent between entering or not, are called the marginal entrants. Since $W_{B}(\underline{v})=W_{S}(\bar{c})=0$, the marginal participating types are equal to the corresponding dynamic types: $\bar{c}=\tilde{c}(\bar{c}), \underline{v}=\tilde{v}(\underline{v})$. Next, since the dynamic types are monotone increasing, we define the sellers' minimum acceptable price $\underline{c}=\tilde{c}(0)$ and the buyers' maximum acceptable price $\bar{v}=\tilde{v}(1)$; taken together they define what we call the acceptance interval $[\underline{c}, \bar{v}]$. From the steady-state mass balance condition $b[1-F(\underline{v})]=s G(\bar{c})$, the marginal participating types $\underline{v}$ and $\bar{c}$ must be on different sides of the Walrasian price $p^{*}$, i.e. either $\bar{c} \leq p^{*} \leq \underline{v}$ or $\underline{v} \leq p^{*} \leq \bar{c}$. Therefore $p^{*}$ must always fall within the acceptance interval, i.e. $p^{*} \in(\underline{c}, \bar{v})$.

\section{The rate of convergence for GRP TIOLI bargaining games}

In this section, we consider bargaining mechanisms whose extensive-form games satisfy two additional assumptions. Call a node a take-it-or-leave-it (TIOLI) node if one of the traders is a TIOLI proposer in that node. Specifically, the proposer offers price $p$, the responder says yes or no, and the bargaining game terminates. The following two assumptions require that both the buyer and the seller can guarantee that they are TIOLI proposers with certain probability.

Assumption 2 (Buyer TIOLI property) The buyer has a strategy $a_{B}$ with the property that regardless of the strategy used by his opponent, the play of the game passes through a node where he is a TIOLI proposer with probability at least $\alpha_{B}>0$.

Assumption 3 (Seller TIOLI property) The seller has a strategy $a_{S}$ with the property that regardless of the strategy used by his opponent, the play of the game passes through a node where she is a TIOLI proposer with probability at least $\alpha_{S}>0$. 
The bargaining mechanisms that satisfy these two additional assumptions will be called generalized random-proposer TIOLI mechanisms, or more succinctly, GRP TIOLI mechanisms.

Our main result in this section is the following theorem and its corollary. We will use the notation $W_{B \tau}, W_{S \tau}, \tilde{v}_{\tau}, \tilde{c}_{\tau}, \bar{v}_{\tau}, \underline{c}_{\tau}, \underline{v}_{\tau}, \bar{c}_{\tau}$ to emphasize the dependence of these equilibrium objects on $\tau$. The theorem provides a lower and upper bounds on the acceptance gap $\bar{v}_{\tau}-\underline{c}_{\tau}$, and the corollary provides an upper bound on the deviations of the equilibrium utilities $W_{B \tau}(v)$ and $W_{S \tau}(c)$ from the Walrasian utilities $W_{B}^{*}(v)$ and $W_{S}^{*}(c)$. The proof of the theorem is rather long and is given in Subsection 4.1.

Theorem 1 For any GRP TIOLI mechanism,

$$
\tau \cdot \kappa \leq \bar{v}_{\tau}-\underline{c}_{\tau} \leq \tau \cdot \frac{K\left(\zeta_{0}\right)}{\alpha_{B}+\alpha_{S}}\left(1+\frac{2 r}{\kappa}\right)^{3} .
$$

where $\kappa \equiv \min \left\{\kappa_{B}, \kappa_{S}\right\}$ and

$$
\zeta_{0} \equiv \frac{\alpha_{B}}{\alpha_{S}} \frac{\kappa_{S}}{\kappa_{B}}
$$

As $\tau \rightarrow 0, \bar{v}_{\tau}-\underline{c}_{\tau} \rightarrow 0$ at a linear rate in $\tau$.

Note that the bounds do not depend on any properties of the distributions $F$ and $G$.

Corollary 1 For any GRP TIOLI mechanism,

$$
\max \left\{\sup _{v \in[0,1]}\left|W_{B}^{*}(v)-W_{B \tau}(v)\right|, \sup _{c \in[0,1]}\left|W_{S}^{*}(c)-W_{S \tau}(c)\right|\right\} \leq \tau \cdot \frac{K\left(\zeta_{0}\right)}{\alpha_{B}+\alpha_{S}}\left(1+\frac{2 r}{\kappa}\right)^{3} .
$$

As $\tau \rightarrow 0$, the rate of convergence is $O(\tau)$.

Proof of Corollary. We only prove the result for the buyers; the proof for the sellers is parallel. Observe that if $v \geq \underline{v}$ then $W_{B \tau}(v)=v-\tilde{v}_{\tau}(v)$; and if $v<\underline{v}$ then $W_{B \tau}(v)=0$. Consequently,

$$
W_{B}^{*}(v)-W_{B \tau}(v)=\max \left\{v-p^{*}, 0\right\}-W_{B \tau}(v)=\left\{\begin{array}{ll}
\tilde{v}_{\tau}(v)-p^{*} & \text { if } v \geq \underline{v}_{\tau} \text { and } v \geq p^{*} \\
\tilde{v}_{\tau}(v)-v & \text { if } v \geq \underline{v}_{\tau} \text { and } v<p^{*} \\
v-p^{*} & \text { if } v<\underline{v}_{\tau} \text { and } v \geq p^{*} \\
0 & \text { if } v<\underline{v}_{\tau} \text { and } v<p^{*}
\end{array} .\right.
$$

In any of the four cases,

$$
\left|W_{B}^{*}(v)-W_{B \tau}(v)\right| \leq \sup _{v \in A_{B}}\left|\tilde{v}_{\tau}(v)-p^{*}\right| \leq \tau \frac{K\left(\zeta_{0}\right)}{\alpha_{B}+\alpha_{S}}\left(1+\frac{2 r}{\kappa}\right)^{3},
$$

where the last inequality follows from $\left|\tilde{v}_{\tau}(v)-p^{*}\right| \leq \bar{v}_{\tau}-\underline{c}_{\tau}$. Q.E.D.

Remark 1 Since $W_{B}^{*}(v)-W_{B \tau}(v)$ and $W_{S}^{*}(c)-W_{S \tau}(c)$ are not guaranteed to be positive, the absolute values are needed. Indeed, if $\underline{v}_{\tau}<p^{*}$, then buyers with type $v \in\left(\underline{v}_{\tau}, p^{*}\right]$ would have strictly positive utilities in equilibrium but have 0 Walrasian utilities. We also do not have a positive lower bound for $\left|W_{B}^{*}(v)-W_{B \tau}(v)\right|$ and $\left|W_{S}^{*}(c)-W_{S \tau}(c)\right|$. Indeed, for some types $v, c \in[0,1]$ we could have $W_{B}^{*}(v)=W_{B \tau}(v)=0, W_{S}^{*}(c)=W_{S \tau}(c)=0$. 
Remark 2 Our explicit bound (14) in Corollary 1 can be tight when frictions are small, even when $\tau$ does not go to 0 . For example, suppose that $\tau=1$ and consider the case of small frictions $\kappa_{B}, \kappa_{S}$ and $r$. For simplicity, suppose that frictions are proportionally small in the following sense: $\left(\kappa_{B}, \kappa_{S}, r\right)=\delta\left(1,1, r_{0}\right)$, where $\delta>0$ is small. Then $r / \kappa=r_{0}$ and

$$
K\left(\zeta_{0}\right)=\delta \cdot\left(\frac{1}{\ell_{B}\left(\zeta_{0}\right)}+\frac{1}{\ell_{S}\left(\zeta_{0}\right)}\right)
$$

where $\zeta_{0}=\alpha_{B} / \alpha_{S}$. Clearly, the r.h.s. of (14) becomes small with $\delta$.

\subsection{Proof of Theorem 1}

Since $W_{B \tau}\left(\underline{v}_{\tau}\right)=W_{S \tau}\left(\bar{c}_{\tau}\right)=0$, recursive equations (5) and (6) imply

$$
\begin{gathered}
\ell_{B}(\zeta) q_{B}\left(\underline{v}_{\tau}\right)\left(\underline{v}_{\tau}-p_{B}\left(\underline{v}_{\tau}\right)\right)=\tau \kappa_{B} \\
\ell_{S}(\zeta) q_{S}\left(\bar{c}_{\tau}\right)\left(p_{S}\left(\bar{c}_{\tau}\right)-\bar{c}_{\tau}\right)=\tau \kappa_{S}
\end{gathered}
$$

where $p_{B}\left(\underline{v}_{\tau}\right)$ (respectively, $p_{S}\left(\bar{c}_{\tau}\right)$ ) is the expected price of the $\underline{v}_{\tau}$-type buyer $\left(\bar{c}_{\tau}\right.$-type seller) conditional on trading. We begin by showing that these conditions imply that the entry gap $\underline{v}_{\tau}-\bar{c}_{\tau}$, if any, is bounded by $K\left(\zeta_{0}\right) /\left(\alpha_{B}+\alpha_{S}\right)$.

Lemma 2 (Bound for entry gap) For any GRP TIOLI bargaining mechanism, we have

$$
\max \left\{\underline{v}_{\tau}-\bar{c}_{\tau}, 0\right\} \leq \tau \cdot \min \left\{\frac{\kappa_{B}}{\alpha_{B} \ell_{B}(\zeta)}, \frac{\kappa_{S}}{\alpha_{S} \ell_{S}(\zeta)}\right\} \leq \tau \cdot \frac{K\left(\zeta_{0}\right)}{\alpha_{B}+\alpha_{S}}
$$

Proof. Recall the buyer TIOLI property, and consider a strategy $a_{B}$ according to which the buyer with type $\underline{v}_{\tau}$ offers $\bar{c}_{\tau}$ in his TIOLI node. This offer is final and is accepted by any seller with $c<\bar{c}_{\tau}$. By the buyer TIOLI property, this $a_{B}$ strategy guarantees him the expected payoff $\alpha_{B} \ell_{B}(\zeta)\left(\underline{v}_{\tau}-\bar{c}_{\tau}\right)$. The equilibrium condition (15) then implies $\kappa_{B} \tau \geq \alpha_{B} \ell_{B}(\zeta)\left(\underline{v}_{\tau}-\bar{c}_{\tau}\right)$. Similarly, we can show that $\kappa_{S} \tau \geq \alpha_{S} \ell_{S}(\zeta)\left(\underline{v}_{\tau}-\bar{c}_{\tau}\right)$, and therefore

$$
\underline{v}_{\tau}-\bar{c}_{\tau} \leq \tau \cdot \min \left\{\frac{\kappa_{B}}{\alpha_{B} \ell_{B}(\zeta)}, \frac{\kappa_{S}}{\alpha_{S} \ell_{S}(\zeta)}\right\}
$$

Now since $\ell_{B}$ is nonincreasing and $\ell_{S}$ is nondecreasing, the above minimum is maximized at $\zeta=\zeta_{0}$ because

$$
\frac{\kappa_{B}}{\alpha_{B} \ell_{B}\left(\zeta_{0}\right)}=\frac{\kappa_{S}}{\alpha_{S} \ell_{S}\left(\zeta_{0}\right)},
$$

and the resulting value of the minimum is $K\left(\zeta_{0}\right) /\left(\alpha_{B}+\alpha_{S}\right)$. Q.E.D.

Lemma 2 implies that, if there is a positive entry gap $\underline{v}_{\tau}-\bar{c}_{\tau}$, its length is $O(\tau)$. For the length of the acceptance interval $\left[\underline{c}_{\tau}, \bar{v}_{\tau}\right]$ we can write:

$$
\begin{aligned}
\bar{v}_{\tau}-\underline{c}_{\tau} & \leq\left[\bar{c}_{\tau}-\underline{c}_{\tau}\right]+\left[\underline{v}_{\tau}-\bar{c}_{\tau}\right]+\left[\bar{v}_{\tau}-\underline{v}_{\tau}\right] \\
& =\int_{0}^{\bar{c}_{\tau}} \tilde{c}_{\tau}^{\prime}(c) d c+\left[\underline{v}_{\tau}-\bar{c}_{\tau}\right]++\int_{\underline{v}_{\tau}}^{1} \tilde{v}_{\tau}^{\prime}(v) d v
\end{aligned}
$$


The idea of the proof of Theorem 1 is to bound the slopes of dynamic types $\tilde{v}_{\tau}^{\prime}(v)$ and $\tilde{c}_{\tau}^{\prime}(c)$. Recall Lemma 1; it implies for a.e. active buyers and sellers

$$
\tilde{v}_{\tau}^{\prime}(v)=\frac{\tau r}{\ell_{B} q_{B}(v)}+o(\tau), \quad \tilde{c}_{\tau}^{\prime}(c)=\frac{\tau r}{\ell_{S} q_{S}(c)}+o(\tau) .
$$

For a given $\zeta$, if the probabilities of trading $q_{B}(v)$ and $q_{S}(c)$ could be bounded from below uniformly for all active types, then it is clear that the slopes $\tilde{v}_{\tau}^{\prime}(v)$ and $\tilde{c}_{\tau}^{\prime}(c)$ would converge to 0 at a linear rate in $\tau$. Unfortunately, we have not found an argument to show this. However, steps 1 and 2 of our proof amount to showing that (i) if $\underline{v}_{\tau} \geq \bar{c}_{\tau}$, the slopes can be bounded for the dynamic types $\tilde{c}_{\tau}(c) \in\left[\underline{c}_{\tau},\left(\underline{c}_{\tau}+\bar{c}_{\tau}\right) / 2\right], \tilde{v}_{\tau}(v) \in\left[\underline{v}_{\tau},\left(\underline{v}_{\tau}+\bar{v}_{\tau}\right) / 2\right]$, and (ii) if $\underline{v}_{\tau}<\bar{c}_{\tau}$, the slopes are bounded for $\tilde{c}_{\tau}(c) \in\left[\underline{c}_{\tau},\left(\underline{c}_{\tau}+\bar{v}_{\tau}\right) / 2\right]$ and $\tilde{v}_{\tau}(v) \in\left[\underline{v}_{\tau},\left(\underline{v}_{\tau}+\bar{c}_{\tau}\right) / 2\right]$.

But, since the lengths of these intervals are respectively $\left(\bar{c}_{\tau}-\underline{c}_{\tau}\right) / 2$ and $\left(\bar{v}_{\tau}-\underline{v}_{\tau}\right) / 2$, this is all that is needed, assuming that $\zeta$ is exogenously given. Step 3 is essentially a tidying-up operation. Step 4 deals with the additional complication that $\zeta$ is endogenous. It is shown that the worst-case value of $\zeta$ is $\zeta_{0}$. The lower bound is established in Step 5 . The intuition for the lower bound is that, for a given $\zeta$, ex-post individual rationality implies that the expected profit of type $\bar{v}_{\tau}$ buyer in a given meeting is no more than $\ell_{B}\left(\zeta_{0}\right)\left(\bar{v}_{\tau}-\underline{c}_{\tau}\right)$ and therefore $\bar{v}_{\tau}-\underline{c}_{\tau} \geq \tau \kappa_{B} / \ell_{B}(\zeta) \geq \tau \kappa_{B}$. Similarly, $\bar{v}_{\tau}-\underline{c}_{\tau} \geq \tau \kappa_{S}$.

We now proceed to the steps of the proof.

Step 1: We claim that

$$
\begin{aligned}
& \text { (a): } \frac{\underline{v}_{\tau}-\underline{c}_{\tau}}{\bar{v}_{\tau}-\underline{c}_{\tau}} \geq \frac{\kappa_{B}}{r+\kappa_{B}} \\
& \text { (b): } \frac{\bar{v}_{\tau}-\bar{c}_{\tau}}{\bar{v}_{\tau}-\underline{c}_{\tau}} \geq \frac{\kappa_{S}}{r+\kappa_{S}} .
\end{aligned}
$$

We provide the proof for part (a) only. The proof for part (b) is the flip of that for part (a). First note that the ex-post individual rationality condition (4) implies $p_{B}\left(\underline{v}_{\tau}\right) \geq \underline{c}_{\tau}$. Since $q_{B}$ is nondecreasing, (15) then implies $\ell_{B} q_{B}(v)\left(\underline{v}_{\tau}-\underline{c}_{\tau}\right) \geq \kappa_{B} \tau$ whenever $v \in\left[\underline{v}_{\tau}, 1\right]$. Then for almost all $v \in\left[\underline{v}_{\tau}, 1\right]$,

$$
\ell_{B} q_{B}(v) \geq \frac{\kappa_{B} \tau}{\underline{v}_{\tau}-\underline{c}_{\tau}}
$$

and therefore

$$
\tilde{v}_{\tau}^{\prime}(v)=\frac{1-R_{\tau}}{1-R_{\tau}+R_{\tau} \ell_{B} q_{B}(v)} \leq \frac{r \tau}{\ell_{B} q_{B}(v)} \leq \frac{r}{\kappa_{B} /\left(\underline{v}_{\tau}-\underline{c}_{\tau}\right)} .
$$

where the second last inequality follows by the concavity of the function $1-e^{-x}$. Hence

$$
\begin{gathered}
\bar{v}_{\tau}-\underline{v}_{\tau}=\int_{\underline{v}_{\tau}}^{1} \tilde{v}_{\tau}^{\prime}(v) d v \leq \frac{r}{\kappa_{B} /\left(\underline{v}_{\tau}-\underline{c}_{\tau}\right)}, \\
\frac{\bar{v}_{\tau}-\underline{v}_{\tau}}{\underline{v}_{\tau}-\underline{c}_{\tau}} \leq \frac{r}{\kappa_{B}}, \\
\frac{1}{\underline{v}_{\tau}-\underline{c}_{\tau}}=\frac{1}{1+\underline{c}_{\tau}}=\frac{\kappa_{B}}{\left.r+\kappa_{\tau}-\underline{v}_{\tau}\right) /\left(\underline{v}_{\tau}-\underline{c}_{\tau}\right)} \geq \frac{1}{1+\frac{r}{\kappa_{B}}}=
\end{gathered}
$$


Step 2: We claim that

$$
\begin{aligned}
& \text { (a): } \min \left\{\underline{v}_{\tau}, \bar{c}_{\tau}\right\}-\underline{c}_{\tau} \leq \frac{\tau 4 r\left(r+\kappa_{B}\right)}{\ell_{S} \alpha_{S} \kappa_{B}} \\
& \text { (b): } \bar{v}_{\tau}-\max \left\{\underline{v}_{\tau}, \bar{c}_{\tau}\right\} \leq \frac{\tau 4 r\left(r+\kappa_{S}\right)}{\ell_{B} \alpha_{B} \kappa_{S}} .
\end{aligned}
$$

Again by symmetry, we only provide a proof for (a). Let $y \equiv \min \left\{\underline{v}_{\tau}, \bar{c}_{\tau}\right\}-\underline{c}_{\tau}$. Consider a type $c$ seller with $\tilde{c}_{\tau}(c) \leq \underline{c}_{\tau}+y / 2$. By the seller TIOLI property, she can guarantee the expected payoff of $\alpha_{S}\left[\underline{v}_{\tau}-\tilde{c}_{\tau}(c)\right]$ by pursuing an $a_{B}$ strategy and offering $\underline{v}_{\tau}$ in her TIOLI node, the offer that is accepted in equilibrium by any buyer with $v>\underline{v}_{\tau}$. Therefore the equilibrium expected payoff in the bargaining game is bounded from below by $\alpha_{S}\left[\underline{v}_{\tau}-\tilde{c}_{\tau}(c)\right]$ :

$$
q_{S}(c)\left[p_{S}(c)-\tilde{c}_{\tau}(c)\right] \geq \alpha_{S}\left[\underline{v}_{\tau}-\tilde{c}_{\tau}(c)\right],
$$

where $p_{S}(c)$ is the expected price conditional on trading. Since $\underline{v}_{\tau}-\tilde{c}_{\tau}(c) \geq \underline{v}_{\tau}-\left(\underline{c}_{\tau}+y / 2\right)$, and our definition of $y$ implies that $y \leq \underline{v}_{\tau}-\underline{c}_{\tau}$, it follows that $\underline{v}_{\tau}-\tilde{c}_{\tau}(c) \geq\left(\underline{v}_{\tau}-\underline{c}_{\tau}\right) / 2$ and therefore

$$
q_{S}(c)\left[p_{S}(c)-\tilde{c}_{\tau}(c)\right] \geq \alpha_{S} \frac{\underline{v}_{\tau}-\underline{c}_{\tau}}{2} .
$$

Since no offer above $\bar{v}_{\tau}$ can be accepted in equilibrium by a buyer, $p_{S}(c) \leq \bar{v}_{\tau}$. Since $\tilde{c}_{\tau}(c)$ is nondecreasing by Lemma 1 , we must also have $\tilde{c}_{\tau}(c) \geq \underline{c}_{\tau}$, and therefore

$$
\begin{aligned}
& q_{S}(c) \geq \frac{\alpha_{S}}{2} \frac{\underline{v}_{\tau}-\underline{c}_{\tau}}{\bar{v}_{\tau}-\underline{c}_{\tau}}, \\
& q_{S}(c) \geq \frac{\alpha_{S} \kappa_{B}}{2\left(r+\kappa_{B}\right)},
\end{aligned}
$$

where the last inequality follows from applying the bound from step 1(a),

Then from (11) in Lemma 1,

$$
\tilde{c}_{\tau}^{\prime}(c)=\frac{1-R_{\tau}}{1-R_{\tau}+R_{\tau} \ell_{S} q_{S}(c)} \leq \frac{r \tau}{\ell_{S} q_{S}(c)} \leq \frac{r \tau}{\ell_{S} \alpha_{S} \kappa_{B} / 2\left(r+\kappa_{B}\right)}=\frac{\tau 2 r\left(r+\kappa_{B}\right)}{\ell_{S} \alpha_{S} \kappa_{B}}
$$

Now we can see that

$$
\frac{y}{2}=\int_{\tilde{c}_{\tau}(c) \in\left[\underline{c}_{\tau}, \underline{c}_{\tau}+\frac{y}{2}\right]} \tilde{c}_{\tau}^{\prime}(c) d c \leq \frac{\tau 2 r\left(r+\kappa_{B}\right)}{\ell_{S} \alpha_{S} \kappa_{B}},
$$

which is the same as (a).

Step 3: Let $\kappa=\min \left\{\kappa_{B}, \kappa_{S}\right\}$. We claim that

$$
\bar{v}_{\tau}-\underline{c}_{\tau} \leq \tau \min \left\{\frac{\kappa_{B}}{\alpha_{B} \ell_{B}}, \frac{\kappa_{S}}{\alpha_{S} \ell_{S}}\right\} \cdot\left(1+\frac{r}{\kappa}\right)\left(1+\frac{2 r}{\kappa}\right)^{2} .
$$

To prove it, first notice that from step 2(a) and (17), we have

$$
\underline{v}_{\tau}-\underline{c}_{\tau}=\min \left\{\underline{v}_{\tau}, \bar{c}_{\tau}\right\}-\underline{c}_{\tau}+\max \left\{\underline{v}_{\tau}-\bar{c}_{\tau}, 0\right\} \leq \frac{\tau 4 r\left(r+\kappa_{B}\right)}{\ell_{S} \alpha_{S} \kappa_{B}}+\frac{\tau \kappa_{S}}{\ell_{S} \alpha_{S}} .
$$


Then from step 1(a),

$$
\begin{aligned}
\bar{v}_{\tau}-\underline{c}_{\tau} & \leq \frac{r+\kappa_{B}}{\kappa_{B}}\left(\underline{v}_{\tau}-\underline{c}_{\tau}\right) \leq \tau \frac{r+\kappa_{B}}{\ell_{S} \alpha_{S} \kappa_{B}}\left[\frac{4 r\left(r+\kappa_{B}\right)}{\kappa_{B}}+\kappa_{S}\right] \\
& =\tau \frac{\kappa_{S}}{\ell_{S} \alpha_{S}}\left(1+\frac{r}{\kappa_{B}}\right)\left[1+\frac{4 r}{\kappa_{S}}\left(1+\frac{r}{\kappa_{B}}\right)\right] \\
& \leq \tau \frac{\kappa_{S}}{\ell_{S} \alpha_{S}}\left(1+\frac{r}{\kappa}\right)\left[1+\frac{4 r}{\kappa}\left(1+\frac{r}{\kappa}\right)\right]=\tau \frac{\kappa_{S}}{\ell_{S} \alpha_{S}}\left(1+\frac{r}{\kappa}\right)\left(1+\frac{2 r}{\kappa}\right)^{2} .
\end{aligned}
$$

Similarly, from step 2(b) and step 1(b),

$$
\bar{v}_{\tau}-\underline{c}_{\tau} \leq \tau \frac{\kappa_{B}}{\ell_{B} \alpha_{B}}\left(1+\frac{r}{\kappa}\right)\left(1+\frac{2 r}{\kappa}\right)^{2} .
$$

$\underline{\text { Step 4: }}$ We get our claim by combining the last two upper bounds for $\bar{v}_{\tau}-\underline{c}_{\tau}$ from the last step:

$$
\bar{v}_{\tau}-\underline{c}_{\tau} \leq \tau \min \left\{\frac{\kappa_{B}}{\ell_{B}(\zeta) \alpha_{B}}, \frac{\kappa_{S}}{\ell_{S}(\zeta) \alpha_{S}}\right\}\left(1+\frac{2 r}{\kappa}\right)^{3} .
$$

Notice that $\kappa_{S} /\left(\ell_{S}(\zeta) \alpha_{S}\right)$ is nonincreasing and $\kappa_{B} /\left(\ell_{B}(\zeta) \alpha_{B}\right)$ is nondecreasing in $\zeta$, and that they are equal if and only if $\zeta=\zeta_{0}$. If $\zeta=\zeta_{0}$, both of them are equal to $K\left(\zeta_{0}\right) /\left(\alpha_{B}+\alpha_{S}\right)$. Thus we obtain the upper bound in the Theorem.

Step 5: Finally, we show that $\bar{v}_{\tau}-\underline{c}_{\tau} \geq \tau \kappa$. Consider once again equations (15) and (16). Since $\ell_{B} q_{B}$ and $\ell_{S} q_{S}$ are at most 1 , and $p_{B}\left(\underline{v}_{\tau}\right) \geq \underline{c}_{\tau}, p_{S}\left(\bar{c}_{\tau}\right) \leq \bar{v}_{\tau}$ by the ex-post individual rationality condition (4), we have

$$
\bar{v}_{\tau}-\underline{c}_{\tau} \geq \tau \kappa_{B}, \quad \bar{v}_{\tau}-\underline{c}_{\tau} \geq \tau \kappa_{S} .
$$

Consequently,

$$
\bar{v}_{\tau}-\underline{c}_{\tau} \geq \tau \max \left\{\kappa_{B}, \kappa_{S}\right\} \geq \tau \kappa
$$

Q.E.D.

\section{Rate of convergence results for general bargaining mech- anisms}

Define

$$
W_{\tau}^{0}=b \int_{A_{B}} W_{B \tau}(v) d F(v)+s \int_{A_{S}} W_{S \tau}(c) d G(c)
$$

as the total welfare of a cohort of buyers and sellers. We show next that, in terms of the total welfare, no bargaining mechanism can attain a faster-than-linear rate of convergence.

Theorem 2 For any bargaining protocol,

$$
W^{0 *}-W_{\tau}^{0} \geq \tau \cdot b\left[1-F\left(\underline{v}_{\tau}\right)\right] \min _{\zeta>0} K(\zeta)
$$

As $\tau \rightarrow 0$, the rate of convergence of $W^{0 *}-W_{\tau}^{0}$ to 0 cannot be faster than linear. 
Proof. One can eliminate $W_{B \tau}(v)$ for $v \in A_{B}$ and $W_{S \tau}(c)$ for $c \in A_{S}$ from the recursive equations (5) and (6):

$$
W_{B \tau}(v)=\frac{\ell_{B}\left[q_{B}(v) v-t_{B}(v)\right]-\tau \kappa_{B}}{1-R_{\tau}+R_{\tau} \ell_{B} q_{B}(v)}, \quad W_{S \tau}(c)=\frac{\ell_{S}\left[t_{S}(c)-q_{S}(c) c\right]-\tau \kappa_{S}}{1-R_{\tau}+R_{\tau} \ell_{S} q_{S}(c)} .
$$

Since the traders in equilibrium enter voluntarily, the numerators in the above fractions are non-negative for $v \in A_{B}$ and $c \in A_{S}$. Consequently, for active types $W_{B \tau}(v)$ and $W_{S \tau}(c)$ can be bounded from above as

$$
\begin{gathered}
W_{B \tau}(v) \leq \frac{\ell_{B}\left[q_{B}(v) v-t_{B}(v)\right]-\tau \kappa_{B}}{\ell_{B} q_{B}(v)} \leq v-\frac{t_{B}(v)}{q_{B}(v)}-\frac{\tau \kappa_{B}}{\ell_{B}}, \\
W_{S \tau}(c) \leq \frac{t_{S}(c)}{q_{S}(c)}-c-\frac{\tau \kappa_{S}}{\ell_{S}} .
\end{gathered}
$$

Now the total welfare of the entering cohort is bounded from above by

$$
\begin{aligned}
& b \int_{A_{B}} v d F(v)-s \int_{A_{S}} c d G(c) \\
& -\left[b \int_{A_{B}} \frac{t_{B}(v)}{q_{B}(v)} d F(v)-s \int_{A_{S}} \frac{t_{S}(c)}{q_{S}(c)} d G(c)\right] \\
& -\left(\frac{\tau \kappa_{B}}{\ell_{B}}+\frac{\tau \kappa_{S}}{\ell_{S}}\right) b \int_{A_{B}} d F(v) .
\end{aligned}
$$

Obviously, the first term does not exceed the Walrasian welfare rate $W_{0}^{*}$. The second term is zero because in a steady state, $b d F(v) / q_{B}(v)=B \ell_{B} d \Phi(v), s d G(c) / q_{S}(c)=S \ell_{S} d \Gamma(c)$ and the transfers are balanced, $B \ell_{B} \int_{v \in A_{B}} t_{B}(v) d \Phi(v)=S \ell_{S} \int_{c \in A_{S}} t_{S}(c) d \Gamma(c)$. Consequently,

$$
W_{\tau}^{0} \leq W^{0 *}-\left(\frac{\tau \kappa_{B}}{\ell_{B}(\zeta)}+\frac{\tau \kappa_{S}}{\ell_{S}(\zeta)}\right) b \int_{A_{B}} d F(v)
$$

The proof is completed by noting that $\tau \kappa_{B} / \ell_{B}(\zeta)+\tau \kappa_{S} / \ell_{S}(\zeta) \geq \tau \cdot \min _{\zeta>0} K(\zeta)$. Q.E.D.

Corollary 2 Any GRP TIOLI mechanism is worst-case asymptotically optimal, in the sense that $W_{\tau}^{0} \rightarrow W^{0 *}$ at a linear rate.

Of course, the intuition for Theorem 2 is that with matching frictions, the loss of welfare due to costly delay is unavoidable regardless of the bargaining protocol. An interesting further question is how to isolate the welfare loss due to strategic behavior. One way of doing so is to compensate the traders for the costs of participation and time discounting that they incur in equilibrium. This will eliminate time discounting and participation costs from the value functions in (19), and we can define the welfare loss due to strategic effects as

$$
\Delta w_{\tau}=W_{\tau}^{0 *}-b \int_{A_{B}}\left(v-\frac{t_{B}(v)}{q_{B}(v)}\right) d F(v)-s \int_{A_{S}}\left(\frac{t_{S}(c)}{q_{S}(c)}-c\right) d G(c) .
$$


Since $b d F(v) / q_{B}(v)=B \ell_{B} d \Phi(v), s d G(c) / q_{S}(c)=S \ell_{S} d \Gamma(c)$, and the transfers are balanced,

$$
B \ell_{B} \int_{v \in A_{B}} t_{B}(v) d \Phi(v)=S \ell_{S} \int_{c \in A_{S}} t_{S}(c) d \Gamma(c),
$$

we have

$$
\Delta w_{\tau}=W^{0 *}-b \int_{A_{B}} v d F(v)+s \int_{A_{S}} c d G(c) .
$$

By Lemma 1, the sets of participating types $A_{B}$ and $A_{S}$ are intervals, $A_{B}=\left[\underline{v}_{\tau}, 1\right]$ and $A_{S}=\left[0, \bar{c}_{\tau}\right]$. The welfare loss $\Delta w_{\tau}$ is then equal to the area of the familiar "deadweight loss" triangle,

$$
\Delta w_{\tau}=b \int_{p^{*}}^{\underline{v}_{\tau}}\left(v-p^{*}\right) d F(v)+s \int_{\bar{c}_{\tau}}^{p^{*}}\left(p^{*}-c\right) d G(c) .
$$

The inefficiency loss $\Delta w_{\tau}$ is due only to inefficient entry, because the marginal participating types $\underline{v}_{\tau}, \bar{c}_{\tau}$ may be different from $p^{*}$. As is well known, the area of the "deadweight loss" triangle is proportional to $\left|\underline{v}_{\tau}-\bar{c}_{\tau}\right|^{2}$, and therefore $\Delta w_{\tau}=O\left(\left|\underline{v}_{\tau}-\bar{c}_{\tau}\right|^{2}\right)$. Theorem 1 implies the following corollary.

Corollary 3 For any GRP TIOLI mechanism, the welfare loss due to strategic behavior $\Delta w_{\tau}=O\left(\tau^{2}\right)$, i.e. converges to 0 at a no-slower-than-quadratic rate.

\section{$6 \quad$ Results for $k$-double auction}

It is interesting to know if convergence, or more strongly convergence at a linear rate, can be proved for bargaining games that do not satisfy the GRP TIOLI property. In this section we show that another well-studied bargaining mechanism, the bilateral $k$-double auction, does not have robust convergence properties. In other words, some equilibria do not converge to perfect competition.

Recall the rules of the bilateral $k$-double auction: the buyer and the seller simultaneously and independently submit a bid price $p_{B}$ and an ask price $p_{S}$ respectively, and then trade occurs if and only if the buyer's bid is at least as high as the seller's ask, at the weighted average price $(1-k) p_{S}+k p_{B}$, where $k \in(0,1)$.

We distinguish two classes of double-auction equilibria: full-trade and non-full-trade. A full-trade equilibrium is characterized by the property that every meeting results in trade. We claim that the class of full-trade equilibria includes equilibria that are very inefficient, even with arbitrarily small frictions. (But it also includes equilibria that converge to perfect competition.)

From Lemma 1 , the sets $A_{B}, A_{S}$ of active types are still intervals $[\underline{v}, 1]$ and $[0, \bar{c}]$ for some marginal types $\underline{v}$ and $\bar{c}$; and we also have $\tilde{v}(v)\langle v$ and $\tilde{c}(c)>c$ for all $v>\underline{v}$ and all $c<\bar{c}$. Since all active traders' trading probabilities are strictly positive, they must in equilibrium submit serious bids/asks, and therefore, we must have $p_{B}(v) \leq \tilde{v}(v)<v$ and $p_{S}(c) \geq \tilde{c}(c)>c$ for all $v>\underline{v}$ and all $c<\bar{c}$. Now it is clear that for an equilibrium to be full-trade, we must have $\bar{c} \leq \underline{v}$, and all traders must submit a common bid/ask $p$. Hence every matched pair trades at the price $p$. Furthermore, $\underline{v}$-buyers and $\bar{c}$-sellers have 
to recover their participation costs, thus in any full-trade equilibrium we have $\bar{c}<p<\underline{v}$ for some $p \in(0,1)$.

Any full-trade equilibrium must satisfy indifference equations for the marginal types, as well as the a mass balance equation:

$$
\begin{aligned}
\ell_{B}(\zeta)(\underline{v}-p) & =\tau \kappa_{B}, \\
\ell_{S}(\zeta)(p-\bar{c}) & =\tau \kappa_{S}, \\
b[1-F(\underline{v})] & =s G(\bar{c}) .
\end{aligned}
$$

The converse is also true, i.e. any quadruple $\{p, \zeta, \underline{v}, \bar{c}\}$ satisfying (20), (21), (22) and $K(\zeta)<1$ must characterize a full-trade equilibrium. In particular, any trader's bestresponse bid/ask strategy is $p$, given that all other active traders use this strategy.

From equations (20) and (21), it follows that the entry gap is

$$
\underline{v}-\bar{c}=\tau K(\zeta) .
$$

The next proposition shows that $\underline{v}-\bar{c}$ can be arbitrary close to 1 for all $\tau$ small enough so that a non-trivial equilibrium exists. Therefore equilibrium outcomes can be arbitrarily far from efficiency even with small frictions. The set of equilibrium entry gaps converges to $(0,1)$ as frictions disappear, so the set of full-trade equilibria ranges from perfectly competitive to almost perfectly inefficient. Moreover, the set of equilibrium prices also converges to $(0,1)$. Thus indeterminacy grows rather than vanishes with competition, contrary to the results in the static double auction literature.

Proposition 1 A full-trade equilibrium exists if and only if

$$
\tau \cdot \min _{\zeta>0} K(\zeta)<1
$$

The set of equilibrium values of $\underline{v}-\bar{c}$ is an interval $\left[\tau \cdot \min _{\zeta>0} K(\zeta), 1\right)$. As $\tau \rightarrow 0$, this set and the set of equilibrium prices converge to $(0,1)$ In particular, there exists a sequence of full-trade equilibria that converges to perfect competition, but also sequences that do not converge.

Proof: The proof follows the graphical argument shown in Figure 1. Given $\tau$, the right panel shows the marginal types $\underline{v}$ and $\bar{c}$ in a steady-state equilibrium. The left panel shows the supportable values of the market tightness $\underline{\zeta}$ and $\bar{\zeta}$ that correspond to the given gap $\underline{v}-\bar{c}<1$. (In general, there can be one, two or more such values.)

Our assumption $M(0, S)=M(B, 0)=0$ implies $\ell_{B}(\infty)=\ell_{S}(0)=0$. It in turn implies

$$
\lim _{\zeta \rightarrow 0} K(\zeta)=\lim _{\zeta \rightarrow \infty} K(\zeta)=\infty,
$$

as depicted in the left panel.

Given that (25) holds, a solution $\zeta$ to the equation $\tau K(\zeta)=\underline{v}-\bar{c}$ exists if and only if $\underline{v}-\bar{c} \in\left[\tau \cdot \min _{\zeta>0} K(\zeta), 1\right)$. Since $\lim _{\tau \rightarrow 0} \tau K(\zeta)=0$ for any $\zeta>0$, we also must have $\tau \cdot \min _{\zeta>0} K(\zeta) \rightarrow 0$ as $\tau \rightarrow 0$. It proves that the set of supportable entry gaps $\underline{v}-\bar{c}$ converges to the interval $(0,1)$. 


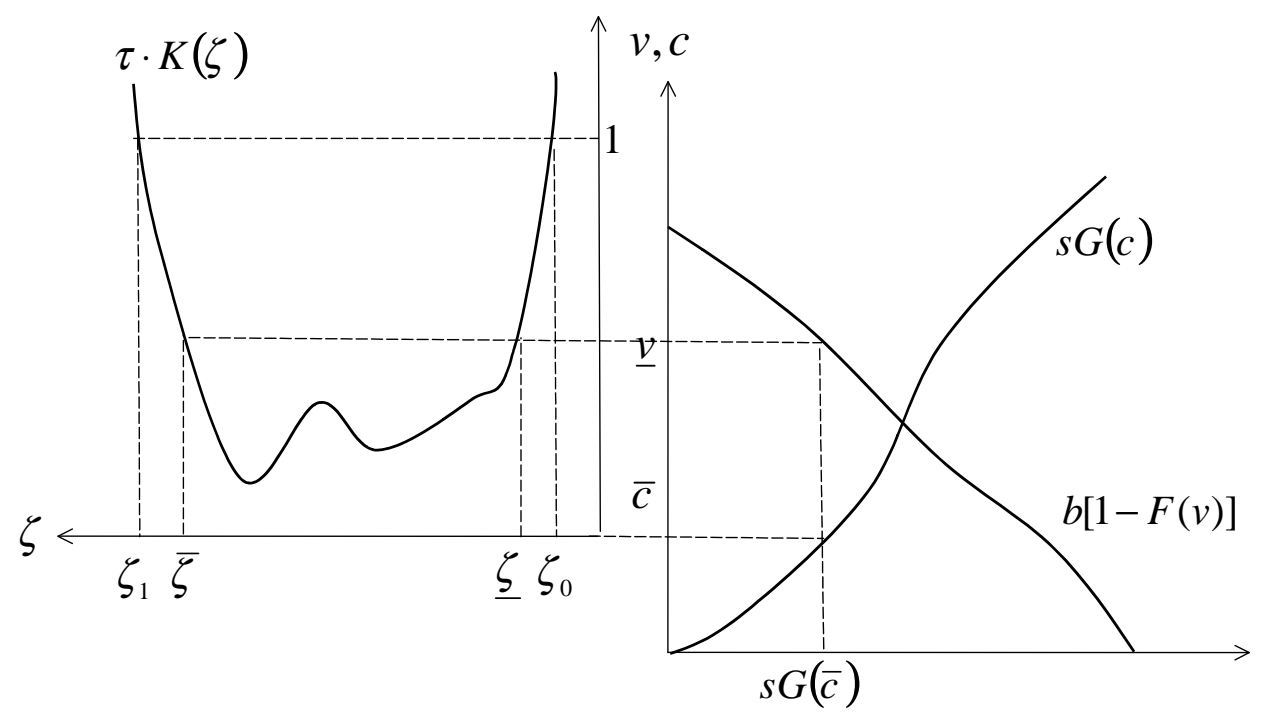

Figure 1: Construction of a full-trade equilibrium of the k-double auction

Now fix any $\tau$ such that $\tau \cdot \min _{\zeta>0} K(\zeta)<1$. Consider the longest interval $\left[\zeta_{0 \tau}, \zeta_{1 \tau}\right]$ such that $\tau K\left(\zeta_{0 \tau}\right)=\tau K\left(\zeta_{1 \tau}\right)=1$ and $\tau K(\zeta)<1$ for $\zeta \in\left(\zeta_{0}, \zeta_{1}\right)$. For any $\zeta \in\left(\zeta_{0 \tau}, \zeta_{1 \tau}\right)$, $\underline{v}$ and $\bar{c}$ can be found uniquely from (23) and (22) (see Figure 1). Denote these mappings as $\underline{v}_{\tau}(\zeta)$ and $\bar{c}_{\tau}(\zeta)$. The equilibrium price $p$ can also be found uniquely from equation (20) or equation (21):

$$
\begin{aligned}
p_{\tau}(\zeta) & \equiv \bar{c}_{\tau}(\zeta)+\frac{\tau \cdot \kappa_{S}}{\ell_{S}(\zeta)} \\
( & \left.=\underline{v}_{\tau}(\zeta)-\frac{\tau \cdot \kappa_{B}}{\ell_{B}(\zeta)}\right) .
\end{aligned}
$$

This formally defines a continuous mapping $p_{\tau}(\cdot)$ of $\left[\zeta_{0 \tau}, \zeta_{1 \tau}\right]$ into $\mathbb{R}_{+}$. Consequently, its image is a closed interval that contains the points $p\left(\zeta_{0 \tau}\right)$ and $p\left(\zeta_{1 \tau}\right)$; and the set of supportable equilibrium prices contains this interval. The definitions of $\zeta_{0 \tau}$ and $\zeta_{1 \tau}$ imply that $\zeta_{0 \tau} \rightarrow 0$ and $\zeta_{1 \tau} \rightarrow \infty$ as $\tau \rightarrow 0$. Now $\bar{c}_{\tau}\left(\zeta_{1 \tau}\right)=0$ for all $\tau$ and $\ell_{S}\left(\zeta_{1 \tau}\right) \rightarrow \infty$ as $\tau \rightarrow 0$, therefore (26) implies that $\lim _{\tau \rightarrow 0} p_{\tau}\left(\zeta_{1 \tau}\right)=0$. Similarly, $\underline{v}_{\tau}\left(\zeta_{0 \tau}\right)=1$ for all $\tau$ and $\ell_{B}\left(\zeta_{0 \tau}\right) \rightarrow \infty$ as $\tau \rightarrow 0$, so that (27) implies that $\lim _{\tau \rightarrow 0} p_{\tau}\left(\zeta_{0 \tau}\right)=1$. This proves that the set of supportable equilibrium price converges to $(0,1)$. Q.E.D.

By looking at the equilibria with the smallest entry gap, $\underline{v}-\bar{c}=\tau \cdot \min _{\zeta>0} K(\zeta)$, we can see that the following corollary is true.

Corollary 4 There are full-trade equilibria that converge, in terms of the ex-ante utilities, at a linear rate in $\tau$.

It is not hard to see that the condition $\tau \cdot \min _{\zeta>0} K(\zeta)<1$ is also necessary for any nontrivial steady-state equilibrium to exist. We thus have the following corollary. 
Corollary 5 There exists a nontrivial steady-state equilibrium (either full-trade or nonfull-trade) if and only if $\tau \cdot \min _{\zeta>0} K(\zeta)<1$.

Proof: Given Proposition 1, it is sufficient to prove the necessity of the condition $\tau \cdot \kappa_{B} / \ell_{B}(\zeta)+\tau \cdot \kappa_{S} / \ell_{S}(\zeta)<1$. The incentive compatibility conditions (2) and (3) imply

$$
\ell_{B}(\zeta) \int[q(v, c) v-t(v, c)] d \Gamma(c) \geq \tau \cdot \kappa_{B}, \quad \ell_{S}(\zeta) \int[t(v, c)-q(v, c) c] d \Phi(v) \geq \tau \cdot \kappa_{S}
$$

and hence

$$
\frac{\tau \cdot \kappa_{B}}{\ell_{B}(\zeta)}+\frac{\tau \cdot \kappa_{S}}{\ell_{S}(\zeta)} \leq \iint(v-c) d \Phi(v) d \Gamma(c)<1
$$

Q.E.D.

Remark 3 This necessary and sufficient condition is weaker than the one for the simple random-proposer TIOLI bargaining, as shown in Shneyerov and Wong (2007).

Proposition 1 shows that, even in the full-trade class, the set of double-auction equilibria is large. Of course, it has been known at least since Chatterjee and Samuelson (1983) and Leininger, Linhart, and Radner (1989) that static double auctions have a huge multiplicity of equilibria. The latter paper in fact looks at full-trade equilibria (which they call one-step equilibria), and shows that such equilibria exist for any price $p \in(0,1)$. (With positive participation costs, only the prices sufficiently far from the endpoints 0 and 1 can be supported.) Our result shows that this multiplicity of equilibria survives in our dynamic model, even as we let the time between meetings go to 0 .

Leininger, Linhart, and Radner (1989) also study $n$-step equilibria, where the strategies are allowed to be $n$-step functions, and also find multiple equilibria in that class. In our setting, these equilibria would be non-full-trade. In the theorem below, we show existence of equilibria with two steps (see Figure 2). ${ }^{12}$ There are two seller cutoff types $\hat{c} \in(0,1)$ and $\bar{c} \in(0,1)$ with $\hat{c}<\bar{c}$, and two buyer cutoff types $\hat{v} \in(0,1)$ and $\underline{v} \in(0,1)$ with $\hat{v}>\underline{v}$. The sellers with $c \in[0, \hat{c})$ enter and bid $p_{S}(c)=p$, where $p$ is some constant strictly below $p^{*}$. The sellers with $c \in[\hat{c}, \bar{c}]$ enter and bid $p_{S}(c)=\bar{p}$, where $\bar{p}>p^{*}$. The sellers with $c \in(\bar{c}, 1]$ do not enter. Similarly, the buyers with $v \in(\hat{v}, 1]$ enter and bid $\bar{p}$, the buyers with $v \in[\underline{v}, \hat{v}]$ enter and submit $\underline{p}$, and the buyers with $v \in[0, \underline{v})$ do not enter.

The following theorem contains our non-convergence result for the two-step class of equilibria.

Theorem 3 For any $a \in(0,1)$, there exist $r_{0}>0, \tau_{0}>0$ and $\bar{W}<W^{0 *}$ such that for all $r \in\left[0, r_{0}\right)$ and $\tau \in\left(0, \tau_{0}\right)$, there exists a two-step equilibrium in which the price spread is larger than a, i.e. $\bar{p}-\underline{p}>a$, and the total ex-ante surplus is smaller than $\bar{W}$, i.e. $W^{0}<\bar{W}$.

\footnotetext{
${ }^{12}$ We don't know if our construction could be generalized to $n$-step equilibria as Leininger, Linhart, and Radner (1989). Generally, this would seem to be a hard problem, especially given that Leininger, Linhart, and Radner (1989) are able to prove existence of an $n$-step equilibrium only for a special case of equal steps. However, equilibria with two steps serve our purpose here, which is to show that there are non-convergent equilibria in the non-full-trade class.
} 


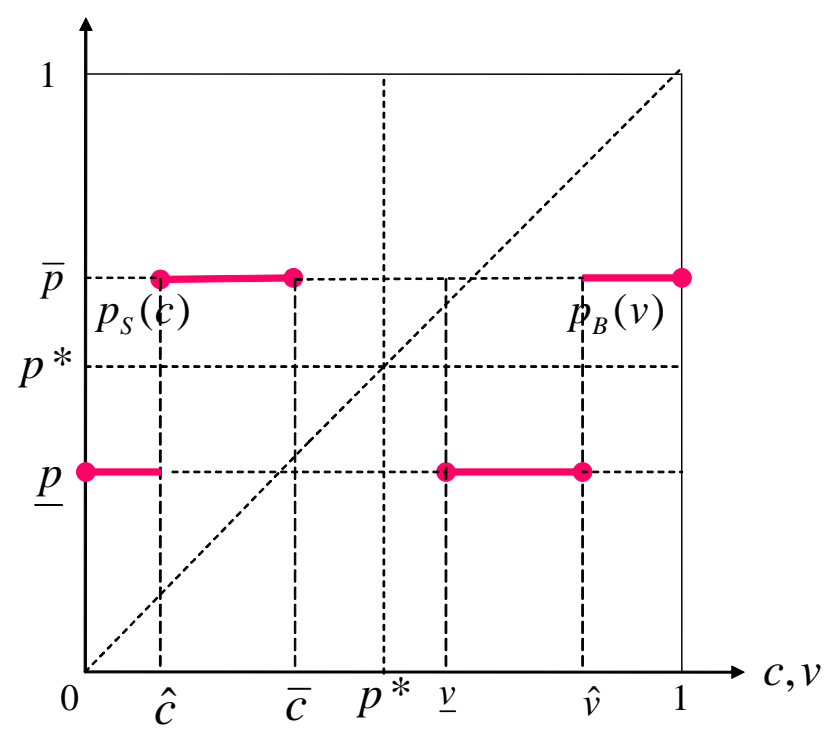

Figure 2: A two-step equilibrium of the $k$-double auction

Proof: We derive a system of equations characterizing the set of two-step equilibria. But before doing so, it is convenient to introduce additional notation. In a two-price equilibrium, the buyers with $v>\hat{v}$ who submit the high bid price $\bar{p}$, trade with any seller they meet. Buyers with $v \in[\underline{v}, \hat{v}]$, who submit the low bid price $p$, trade only with sellers having $c<\hat{c}$, who submit $p$; their probability of trading is equal to $\Gamma(\hat{c})$. Similarly sellers with $c<\hat{c}$ trade with any buyer they meet, and sellers with $c \in[\hat{c}, \bar{c}]$ trade only with those buyers with $v>\hat{v}$; their probability of trading is equal to $1-\Phi(\hat{v})$.

In the equilibria to be constructed $\Gamma(\hat{c})$ and $1-\Phi(\hat{v})$ will converge to 0 as $\tau$ goes to 0 , so it is convenient to divide them by $\tau$ :

$$
\pi_{B} \equiv \frac{1-\Phi(\hat{v})}{\tau}, \quad \pi_{S} \equiv \frac{\Gamma(\hat{c})}{\tau} .
$$

Since $\underline{v}$-buyers and $\bar{c}$-sellers are indifferent between entering or not, we have

$$
\begin{aligned}
& \ell_{B} \pi_{S}(\underline{v}-\underline{p})=\kappa_{B}, \\
& \ell_{S} \pi_{B}(\bar{p}-\bar{c})=\kappa_{S} .
\end{aligned}
$$

Since $\hat{v}$-buyers are indifferent between biding $\underline{p}$ or $\bar{p}$, and $\hat{c}$-sellers are indifferent between asking $\underline{p}$ or $\bar{p}$, we have

$$
\begin{aligned}
& \tau \pi_{S}[\tilde{v}(\hat{v})-\underline{p}]=\tau \pi_{S}\{\tilde{v}(\hat{v})-[(1-k) \underline{p}+k \bar{p}]\}+\left(1-\tau \pi_{S}\right)[\tilde{v}(\hat{v})-\bar{p}], \\
& \tau \pi_{B}[\bar{p}-\tilde{c}(\hat{c})]=\tau \pi_{B}\{[(1-k) \underline{p}+k \bar{p}]-\tilde{c}(\hat{c})\}+\left(1-\tau \pi_{B}\right)[\underline{p}-\tilde{c}(\hat{c})] .
\end{aligned}
$$

Since the utility equations (8), (9) still hold here, we have

$$
\hat{W}_{B}=(\hat{v}-\underline{v}) \frac{m(\zeta) \pi_{S}}{\zeta\left(1-R_{\tau}\right)+R_{\tau} m(\zeta) \pi_{S}}
$$




$$
\hat{W}_{S}=(\bar{c}-\hat{c}) \frac{m(\zeta) \pi_{B}}{1-R_{\tau}+R_{\tau} m(\zeta) \pi_{B}} .
$$

where we denoted $\hat{W}_{B} \equiv W_{B}(\hat{v})$ and $\hat{W}_{S} \equiv W_{S}(\hat{c})$.

To complete the description of a two-step equilibrium, the indifference conditions are supplemented with steady-state mass balance conditions for each interval of types. Here, it suffices to require that the total inflows into the intervals $[\underline{v}, 1]$ and $[0, \bar{c}]$ are balanced with the outflows,

$$
\begin{gathered}
b[1-F(\underline{v})]=S m(\zeta)\left[\pi_{S}+\pi_{B}\left(1-\tau \pi_{S}\right)\right], \\
s G(\bar{c})=S m(\zeta)\left[\pi_{B}+\pi_{S}\left(1-\tau \pi_{B}\right)\right],
\end{gathered}
$$

and that the inflows into the intervals $v \in[\hat{v}, 1]$ and $[0, \hat{c}]$ are also balanced with the outflows,

$$
\begin{gathered}
b[1-F(\hat{v})]=S m(\zeta) \pi_{B}, \\
s G(\hat{c})=S m(\zeta) \pi_{S} .
\end{gathered}
$$

We also define the price spread,

$$
a_{0} \equiv \bar{p}-\underline{p} .
$$

Then equations (28) through (37) form a 10-equation system with 12 endogenous variables $\left\{p, a_{0}, \zeta, \underline{v}, \bar{c}, \hat{v}, \hat{c}, \pi_{B}, \pi_{S}, S, \hat{W}_{B}, \hat{W}_{S}\right\}$. This system does characterize an equilibrium. Indeed, one can easily see that the buyers with $v \in(\hat{v}, 1]$ strictly prefer to bid $\bar{p}$, the buyers with $v \in(\underline{v}, \hat{v})$ strictly prefer to bid $p$, and the buyers with $v \in[0, \underline{v})$ strictly prefer not to enter. Similar remark applies for sellers.

Since we have two degrees of freedom, we can fix some $\zeta>0$ and $a_{0} \in(a, 1)$ and then let equations (28) - (37) determine $\left\{\underline{p}, \underline{v}, \bar{c}, \hat{v}, \hat{c}, \pi_{B}, \pi_{S}, S, \hat{W}_{B}, \hat{W}_{S}\right\}$. We claim that a solution exists for small enough $\tau$ and $r$. To see this, one can check that when $\tau=r=0$, we have a (unique) solution with $\underline{p}$ implicitly determined by $b\left[1-F\left(\underline{p}+a_{0}\right)\right]=s G(\underline{p})$, and all other variables given by

$$
\begin{gathered}
\bar{c}=\underline{p}, \quad \underline{v}=\bar{p}=\underline{p}+a_{0}, \quad \pi_{B}=\frac{\kappa_{S}}{m(\zeta) a_{0}}, \quad \pi_{S}=\frac{\kappa_{B} \zeta}{m(\zeta) a_{0}}, \quad S=\frac{s G(\underline{p}) a_{0}}{\kappa_{B} \zeta+\kappa_{S}}, \\
1-F(\hat{v})=\frac{[1-F(\bar{p})] \kappa_{S}}{\kappa_{B} \zeta+\kappa_{S}}, \quad G(\hat{c})=\frac{G(\underline{p}) \kappa_{B} \zeta}{\kappa_{B} \zeta+\kappa_{S}}, \quad \hat{W}_{B}=\hat{v}-\bar{p}, \quad \hat{W}_{S}=\underline{p}-\hat{c} .
\end{gathered}
$$

One can also check that the Jacobian evaluated at $\tau=r=0$ is not zero. ${ }^{13}$ Therefore the Implicit Function Theorem applies. Because $\bar{p}-p \equiv a_{0}>a$, there exists a two-step equilibrium with $\bar{p}-\underline{p}>a$ when $\tau$ and $r$ are small enough. Moreover, since $\underline{v} \rightarrow \bar{p}$ and $\bar{c} \rightarrow \underline{p}$ as $(\tau, r) \rightarrow(0,0)$, the spread $\underline{v}-\bar{c}$ is also bounded below by $a$. It follows that the associated total ex-ante surplus $W^{\overline{0}}$ is bounded away from the Walrasian total ex-ante surplus $W^{0 *}$. Q.E.D.

Unlike in Proposition 1, the construction in the proof of Theorem 3 treats buyers and sellers symmetrically. In particular, $\zeta$ could be fixed at any value. Then why does the double

\footnotetext{
${ }^{13}$ The Mathematica ${ }^{\circledR}$ notebook that contains the evaluation of the Jacobian is available at artyom239.googlepages.com.
} 
auction mechanism has non-Walrasian limit equilibria while the GRP TIOLI mechanisms do not? One can verify that the dynamic types do collapse to singletons even in the twostep non-convergent equilibria. For the sake of simplicity, suppose the discount rate $r$ is 0 so that the dynamic types are constant and equal $\tilde{c}=\bar{c} \rightarrow \underline{p}$ and $\tilde{v}=\underline{v} \rightarrow \bar{p}$. Also suppose $\tau$ is very small. Then all buyers have dynamic types approximately equal to $p$ and all sellers have dynamic types approximately $\bar{p}$. A seller submitting an ask lower than the dynamic types of all buyers does not guarantee herself a successful trade. To guarantee a trade, she has to ask lower than all buyers' bids. Consider a seller with $c<\hat{c}$. This seller's equilibrium ask price is $\underline{p}$. She realizes that the buyers' dynamic valuations $\tilde{v}$ are always $\bar{p}$ approximately, and would like to demand that much if she could be sure that her offer would be accepted, as it would be in a TIOLI node of the GRP TIOLI bargaining game. However, demanding that much now runs into the risk of being countered with the buyer's bid of $p$, resulting in no trade. In our equilibrium with $\tau$ small, most of the active buyers bid $\underline{p}$. Weighing these trade-offs, the seller decides to submit $\underline{p}$ and not $\bar{p}$. Similar logic applies to the buyers.

Now consider a seller with $c=\underline{p}+\varepsilon$ where $\varepsilon>0$ is small. Although her type (or dynamic type) is significantly lower than the buyers' dynamic types, which are $\bar{p}$ approximately, she chooses not to enter even when the expected participation costs incurred until the next meeting are very small as $\tau$ becomes very small. This is again because most of the active buyers bid $\underline{p}$, making her prospect of trade meager. Similar logic applies to the buyers.

Finally, to complete our logic, we explain why the fraction of active buyers bidding $\underline{p}$ is very high relative to the fraction bidding $\bar{p}$. It is because in our equilibrium, the buyers who bid $p$ can only trade with those sellers who ask $p$, which makes their outflow rate tiny. On the other hand, the buyers who bid $\bar{p}$ trade in any meeting. Thus in the steady state, the buyers who bid $p$ accumulate and dominate the buyers' side of the market. Similar logic applies to the sellers. Taken together, these arguments explain why extra-marginal traders do not enter to quest a fraction of the unexploited surplus $\underline{v}-\bar{c}$, keeping a positive gap between $\underline{v}$ and $\bar{c}$.

The rules of the double auction do not provide a tight connection between the dynamic types and actual acceptance levels as would be the case in the TIOLI nodes of GRP TIOLI mechanisms. Here, a bid/ask is both an offer and an acceptance level. On the other hand, in the TIOLI nodes of GRP TIOLI mechanisms, the proposing and responding strategies are separate decisions because traders are clear about who is a proposer and who is a responder. Ex-post, the bargaining power is given to one party, and thus well-defined. Therefore the responder is always held to her acceptance level, which by the buyer and seller TIOLI properties, creates strong incentive to enter for both buyers and sellers. This drives the marginal participating types close to each other and at the same time close to the Walrasian price.

\section{Concluding remarks}

A limitation of our models is that we assume continua of traders. While this assumption is standard in many matching and bargaining models, it would be nice to have a theorem showing that the continuum economy is a limit, in an appropriate sense, of an economy with 
a finite number of agents. ${ }^{14}$ For example, one might assume that the number of newborn buyers is approximately $n \cdot b$ and the number of newborn sellers is approximately $n \cdot s$, where $n$ is a large integer. Therefore ideally, the setting in this paper would be better viewed as the one of double limits, letting first the number $n$ go to infinity, and then letting $\tau$ go to 0 . Although appealing, we conjecture that such a connection is unlikely to be established at the level of generality of our model, and would need to be established on a case-by-case basis, for more narrowly defined classes of bargaining mechanisms. One reason for this is that we would need first establish the existence of a nontrivial market equilibrium. The issue here is that a trivial equilibrium with no entry always exists. Clearly, when the participation costs are large, this is the only equilibrium. Then how small do the participation costs need to be so that there is a nontrivial equilibrium? For a general bargaining game, there may not be a clear answer to this question.

For simple random-proposer TIOLI games, Shneyerov and Wong (2007) derive a simple necessary and sufficient condition for the existence of a nontrivial market equilibrium. ${ }^{15}$ We conjecture that a formal link between finite $n$ and continuum models might be possible to establish for this class of protocols. A number of hurdles would need to be overcome. The main difficulty is that the type of a trader would include not only his valuation, but also his market history, which leads to multidimensional type spaces. (For example, a buyer who was unable to trade for quite a few periods would infer that the distribution of seller types in the market is tilted towards higher costs, and would adjust his bargaining strategy accordingly.) This difficulty could be overcome if the market type distributions are observable. With this assumption, the market distributions can be viewed as a state variable, and one can define a Markov perfect equilibrium. One important difference from our steady-state continuum model is that the time path of the economy is not in a steady state. So one way to proceed would be to fix an equilibrium $E$ of the continuum model, and to assume that the initial distributions of types are samples of sizes approximately $n \cdot B$ and $n \cdot S$ from $\Phi$ and $\Gamma$. In other words, the economy is jump-started essentially in the steady-state of the continuum model. We conjecture that one can select a sequence of finite- $n$ equilibria $\left\{E_{n}\right\}$ whose equilibrium objects' time paths converge in probability, and in an appropriate sense, to the steady-state time path of $E$.

Our GRP TIOLI mechanisms are restricted to bilateral bargaining. Generalizing our results to multilateral bargaining in a market with many-to-many matches as e.g. in Dagan, Serrano, and Volij (2000) would be important. A different issue is that our non-convergent examples for the $k$-double auction are all quite special since they require a great deal of coordination among the traders. Additional assumptions, e.g. continuity of strategies, could be imposed to restrict the set of equilibria with the purpose of proving their convergence at a linear rate. In addition, allowing a multilateral matching technology might also restore

\footnotetext{
${ }^{14}$ One approach to this problem is taken in McAfee (1993), a paper that studies a dynamic matching environment in which each seller runs an auction among the buyers she is matched with. McAfee (1993) defines a competitive subform consistent equilibrium (CSCE). However, a CSCE does not involve an optimal agent behavior in the finite environment. The convergence result in Peters (2000) is the only theorem we could find that shows a link between exact equilibria in finite and continuum environments. However, his model is essentially a static, directed search model, in which most of the dynamic complications of our setting do not arise.

${ }^{15}$ Their model is set up in continuous time, but it is clear how their result would carry over to a discrete time setting.
} 
convergence of all equilibria of the $k$-double auction mechanism. These extensions are left for future research.

\section{References}

Atakan, A. (2008): "Competitive Equilibria in Decentralized Matching with Incomplete Information," Working paper, Northwestern University.

Ausubel, L., P. Cramton, and R. Deneckere (2002): "Bargaining with Incomplete Information," Handbook of Game Theory, 3, 1897-1945.

Blouin, M., and R. Serrano (2001): "A Decentralized Market with Common Values Uncertainty: Non-Steady States," Review of Economic Studies, 68(2), 323-346.

Butters, G. (1979): "Equilibrium price distributions in a random meetings market," Working paper.

Chatterjee, K., and W. Samuelson (1983): "Bargaining under Incomplete Information," Operations Research, 31(5), 835-851.

Cripps, M., And J. Swinkels (2005): "Efficiency of Large Double Auctions," Econometrica, 74(5), 47-92.

Dagan, N., R. Serrano, and O. Voliu (2000): "Bargaining, coalitions and competition," Economic Theory, 15, 279-296.

De Fraja, G., and J. Sakovics (2001): "Walras Retrouve: Decentralized Trading Mechanisms and the Competitive Price," Journal of Political Economy, 109(4), 842-863.

Gale, D. (1986): "Bargaining and Competition Part I: Characterization," Econometrica, 54(4), 785-806.

- (1987): "Limit Theorems for Markets with Sequential Bargaining," Journal of Economic Theory, 43(1), 20-54.

Gresik, T., and M. Satterthwaite (1989): "The Rate at which a Simple Market Becomes Efficient as the Number of Traders Increases: an Asymptotic Result for Optimal Trading Mechanisms," Journal of Economic Theory, 48, 304-332.

Lauermann, S. (2006a): "Dynamic Matching and Bargaining Games: A General Approach," Working paper, University of Bonn. Bonn.

(2006b): "Efficiency of Decentralized Trading," Working paper, University of (2008): "Price Setting in a Decentralized Market and the Competitive Outcome," Working Paper, University of Michigan. 
Leininger, W., P. Linhart, and R. Radner (1989): "Equilibria of the sealed-bid mechanism for bargaining with incomplete information," Journal of Economic Theory, $48(1), 63-106$.

McAfee, R. (1993): "Mechanism Design by Competing Sellers," Econometrica, 61(6), $1281-1312$.

Milgrom, P., And I. Segal (2002): "Envelope Theorems for Arbitrary Choice Sets," Econometrica, 70(2), 583-601.

Moreno, D., And J. Wooders (2002): "Prices, Delay, and the Dynamics of Trade," Journal of Economic Theory, 104(2), 304-339.

Mortensen, D., and R. Wright (2002): "Competitive Pricing and Efficiency In Search Equilibrium," International Economic Review, 43(1), 1-20.

Peters, M. (2000): "Limits of Exact Equilibria for Capacity Constrained Sellers with Costly Search," Journal of Economic Theory, 95(2), 139-168.

Reny, P., And M. Perry (2006): "Toward a Strategic Foundation for Rational Expectations Equilibrium," Econometrica, 74(3), 1231-1269.

Rubinstein, A., And A. Wolinsky (1985): "Equilibrium in a Market with Sequential Bargaining," Econometrica, 53(5), 1133-1150.

(1990): "Decentralized Trading, Strategic Behaviour and the Walrasian Outcome," The Review of Economic Studies, 57(1), 63-78.

Rustichini, A., M. Satterthwaite, and S. Williams (1994): "Convergence to Efficiency in a Simple Market with Incomplete Information," Econometrica, 62(5), 10411063 .

Satterthwaite, M. (1989): "Bilateral trade with the sealed bid k-double auction: Existence and efficiency.," Journal of Economic Theory, 48(1), 107-133.

Satterthwaite, M., And A. Shneyerov (2007): "Dynamic Matching, Two-sided Incomplete Information, and Participation Costs: Existence and Convergence to Perfect Competition," Econometrica, 75(1), 155-200.

(2008): "Convergence to Perfect Competition of a Dynamic Matching and Bargaining Market with Two-sided Incomplete Information and Exogenous Exit Rate," Games and Economic Behavior, 63(2), 435-467.

Satterthwaite, M., and S. Williams (1989): "The Rate of Convergence to Efficiency in the Buyer's Bid Double Auction as the Market Becomes Large," The Review of Economic Studies, 56(4), 477-498.

(2002): "The Optimality of a Simple Market Mechanism," Econometrica, 70(5), $1841-1863$. 
Serrano, R. (2002): "Decentralized Information and the Walrasian Outcome: A Pairwise Meetings Market with Private Values," Journal of Mathematical Economics, 38(1), 6589.

Shneyerov, A., And A. Wong (2007): "Bilateral Matching and Bargaining with Private Information," Working Paper, UBC.

TAtur, T. (2005): "On the Trade off Between Deficit and Inefficiency and the Double Auction with a Fixed Transaction Fee," Econometrica, 73(2), 517-570.

Williams, S. (1991): "Existence and Convergence of Equilibria in the Buyer's Bid Double Auction," The Review of Economic Studies, 58(2), 351-374.

Wolinsky, A. (1988): "Dynamic Markets with Competitive Bidding," The Review of Economic Studies, 55(1), 71-84.

(1990): "Information Revelation in a Market with Pairwise Meetings," Econometrica, 58(1), 1-23. 